\title{
Evaluation of microbial content of some sunscreen creams in Iran's market
}

\author{
Narges Sedghi Sharif-Abad ${ }^{1}$, Majid Saeedi ${ }^{2,3 *}$, Reza Enayatifard ${ }^{2}$, Katayoun Morteza-Semnani ${ }^{4}$, \\ Jafar Akbari ${ }^{2}$ \\ ${ }^{1}$ Student Research Committee, Mazandaran University of Medical Sciences, Sari, Iran \\ ${ }^{2}$ Department of Pharmaceutics, Faculty of Pharmacy, Mazandaran University of Medical Sciences, Sari, Iran \\ ${ }^{3}$ Pharmaceutical Sciences Research Centre, Mazandaran University of Medical Sciences, Sari, Iran \\ ${ }^{4}$ Department of Medicinal Chemistry, Faculty of Pharmacy, Mazandaran University of Medical Sciences, Sari, Iran
}

\begin{abstract}
The risk of microbial contamination in the cosmetic products especially in smuggled preparations and transmission of it to consumers is very high. In this study, the microbial content and the pollution of some sunscreen creams in the market and one sample in official market as witness were evaluated. The microbial content (bacterial total count, fungal count, and presence of Pseudomon asaeroginosa, Staphylococcus aureus and Entrobacter) of 5 samples of sunscreen cream in the market and two samples in official market were evaluated by two methods (pour plate and Multiple tube technique). All samples showed high microbial and fungal contamination. Entrobacters was observed in all samples. Staphylococcus aureus was recognized in one of the non-standard sunscreens. High level of contamination in sunscreen creams, can affect consumers, health. It seems that low grade raw materials, and insufficient manufacturing surveillance in production process are the main factors in the contamination.
\end{abstract}

Keywords: Cosmetic, sunscreen, microbial content, Pseudomonas aeroginosa, Staphylococcus aureus

Pharm Biomed Res 2015; 1(2): 30-34～DOI: 10.18869/acadpub.pbr.1.2.30

\section{Introduction}

Cosmetic products should be easy to use, effective, and especially safe. Microbial contamination in cosmetic products will be follow risk of infection of consumer and due to extensive uses and no standardization of some cosmetics, possibility of transferring contamination and risk of infections by types of bacteria not unexpected and from the perspective of health at the community level is important $(1,2)$. Skin is the first barrier for body protection. Skin due to is sensitive and very vulnerable, using of suitable sunscreens is the most common way of skin protection in front of direct sunlight (3).
Sunscreens are not sterile products but according to the topical application, those must have the necessary microbial criteria. Preservatives are used to prevent growing of microorganisms in water containing product during use. They must be free of pathogen microorganisms and total number of aerobic microorganism per gram or milliliter must be in pharmacopeia criteria (3-7). Microbial counts below $500 \mathrm{CFU} / \mathrm{g}$ for eye-area product and $1000 \mathrm{CFU} / \mathrm{g}$ for non-eye-area products are accepted in United States Pharmacoepeia (8). Based on these criteria, Staphylococcus aureus and 
Pseudomonas aeroginosa should not be observed in these products (9).

The cosmetic products may be spoiled in two ways: in manufacturing process or by during consumer use (8). Raw materials and manufacturing apparatus are the main sources for microbial contaminantion. This contamination might be causes changes in the composition, odor, or color of the products $(8,10)$.

Contamination of cosmetic products directly may affects on human health as a result of the formation of metabolites microbial harmful and spoilage of products (3). Legislation and introduction of GMP (Good Manufacturing Practice) has improved the microbiological standards, but a contaminated cosmetic product has serious consequences for the consumers (8). By use of water and raw material with suitable quality, GMP leads to preparation of products with lower microbial contamination. The suitable storage and the use of noninvasive packages (such as tubes, pumps or narrow orifice containers) causes of reducing contamination levels during storage and using products remains (11). The inclusion of essential minerals, growth factors, moisture content and acidity provides favorable environment for microbial growth (12).

Only a few studies have been done on the microbial quality of sunscreens and there are very limited data available in Iran. The present study was performed to determine the microbial quality of sunscreens products (illegal and non-ilegal products) available in Iran market.

\section{Materials and methods}

In this study, the microbial content of seven sunscreen creams (five foreign creams from informal and non-standard market and two creams as control from Iranian products which are presented drugstore) was evaluated.
All of the samples were analyzed to detect the presence of total bacterial and fungal count (yeast and mold). The presence of Staphylococcus aureus, Pseudomonas aeroginosa, and Enterobacters were investigated based on United States Pharmacopeia (13). The surface of three sample containers from each cream were swabbed and disinfected by $70 \%$ (v/v) ethanol before opening. For tubed products a large sample was extruded into a sterile plate and mixed thoroughly with a sterile spatula. Samples were opened and weighed under the laminar air flow with aseptic conditions. To determine the microbial content of the samples and ensure the absence of antimicrobial effect of potential products and the possibility of microorganisms growth, preliminary experiment with inoculation of Staphylococcus aureus (PTCC 1112) and Pseudomonas aeroginosa (PTCC 1074) were performed on all samples.

Lack of growth of inoculated microorganisms on the plates, showed the action of preservative and was proved necessityof the use of 3\% Tween 80 and $0.5 \%$ lecithin for neutralization of preservative effect. Pour plate and multipletube methods were used for total microbial counts. In the Plate Method, one gram was aseptically taken from each product and placed into a 9-ml sterile normal saline solution as diluents with $3 \%$ Tween 80 and $0.5 \%$ lecithin thus giving a $10^{-1}$ dilution. Bacterial challenge levels and yeast and mold were $10^{4} \mathrm{CFU} / \mathrm{g}$ of products.

One milliliter of above suspension was transferred into two sterile plates, under aseptic conditions. For aerobic bacterial colony counts, 20 milliliter of sterile Soybean Casein Digest Agar (SCDA) (Merck Co, Germany) was added at $45{ }^{\circ} \mathrm{C}$ to each plate aseptically. The plates were rotated to completely disperse and then incubated at $35{ }^{\circ} \mathrm{C}$ for $24-48$ h. Sabouraud 
Dextrose Agar (SDA) (Merck Co, Germany) was used for yeast and mold cultivation, and incubated at $25{ }^{\circ} \mathrm{C}$ for $5-7$ days. After incubation, the number of colonies was recorded for each plate.

In the multiple-tubes method, one gram was aseptically taken from each product and placed into a 9-mL sterile normal saline solution as diluents with 3\% Tween 80 and $0.5 \%$ lecithin. One milliliter of this suspension was transferred into tubes containing Soybean Casein Digest Broth (SCDB) (Merck Co, Germany) according to the method, aseptically and then were incubated at $35^{\circ} \mathrm{C}$ for $48 \mathrm{~h}$ than tubes with no growth were reported negative for the presence of microorganisms.

For detecting prohibited microorganisms according to the USP, one gram was taken from each product under condition aseptic and placed into a $10-\mathrm{ml}$ sterile normal saline solution as diluent with $3 \%$ Tween 80 and $0.5 \%$ lecitin and was mixed well and tubes were incubated at $35^{\circ} \mathrm{C}$ for $48 \mathrm{~h}$. After incubation, tubes with no growth were reported negative for the presence of Staphylococcus aureus, Pseudomonas aeroginosa and Enterobacters, then a loop of medium with positive growth was transferred to Mannitol-salt Agar (Merck Co, Germany) medium for Staphylococcus aureus detection. This process was performed to Cetrimide Agar and Mac. conkey agar (Merck Co, Germany) for Pseudomonas aeroginosa and Entrobacter detection respectively. After incubation at $35{ }^{\circ} \mathrm{C}$ for $48 \mathrm{~h}$, the plates were checked. In the case of growth on these selective mediums, the sample was reported positive for the presence of related microorganism.

\section{Results}

The results of the plate method showed that microbial count in $60 \%$ (3 creams) of the non-standard samples and all of control samples contamination was more than
$10^{4} \mathrm{CFU} / \mathrm{g}$ (Table 1). The results of total count of all samples based on multiple tube method showed that all of samples were outside the standard range (contamination $>10^{3} \mathrm{CFU} \cdot \mathrm{g}^{-1}$ or $\left.\mathrm{mL}^{-1}\right)$.

Fungal (yeast and mold) contamination was observed in all of sunscreens and total count was more than $10^{3} \mathrm{CFU}_{\mathrm{g}}{ }^{-1}$ or $\mathrm{mL}^{-1}$. Enterobacter contamination was observed in all samples and in $14.29 \%$ ( 1 of 7 creams) of cases but Staphylococcus aureus contamination was seen only in one of the non-standard sunscreen cream. Pseudomonas aeroginosa was not observed in these preparations.

\section{Discussion}

Hygienic characteristics of creams and other cosmetic products to provide the consumer with regard to the standards defined in each country should be at an acceptable level. Condition of manufacturing, GMP observation and antimicrobial preservative are the main factors in microbial contamination and level of hygienic state of cosmetics and toiletries, according to criteria of National Institute of Standards (14). Other researches proved that one of the main recall reasons is microbial contamination of cosmetic products (3-5).

Non-compliance with hygiene issues in the manufacturing processes of product and hygienic products with packaging nonstandard and inadequate can provide easily background of microbial contamination of product. Staphylococcus aureus was one of pathogenic bacteria isolated in this study that according to the USP should not be seen in topical preparations. It is from the most factors important skin pathogens that can gain antibiotic resistance genes through chromosomal and non-chromosomal multiple ways and causing is irreparable effects on consumers (14). 
Table 1 The results of total bacterial and fungal count, and presence of forbidden microorganism

\begin{tabular}{|c|c|c|c|c|}
\hline \multirow{2}{*}{$\begin{array}{l}\text { Sunscreen } \\
\text { cream code }\end{array}$} & \multicolumn{2}{|c|}{ Total bacterial count } & Total & \multirow[b]{2}{*}{ Isolated microorganisms } \\
\hline & $\begin{array}{r}\text { Plate method } \\
\text { (CFU /g) }\end{array}$ & $\begin{array}{r}\text { Multiple-tube } \\
\text { method }\end{array}$ & $\begin{array}{r}\text { yeast and mold } \\
\text { counts }(\text { CFU /g) }\end{array}$ & \\
\hline Sample 1 & $1 \times 10^{2}$ & $>1100$ & $3 \times 10^{4}$ & Enterobacter \\
\hline Sample 2 & $6 \times 10^{2}$ & $>1100$ & $3 \times 10^{3}$ & Enterobacter \\
\hline Sample 3 & $1 \times 10^{5}$ & $>1100$ & $6 \times 10^{4}$ & $\begin{array}{l}\text { Enterobacter, } \\
\text { Staphylococcus aureus }\end{array}$ \\
\hline Sample 4 & $2 \times 10^{4}$ & $>1100$ & $1 \times 10^{4}$ & Enterobacter \\
\hline Sample 5 & $4 \times 10^{4}$ & $>1100$ & $1 \times 10^{4}$ & Enterobacter \\
\hline Control 1 & $6 \times 10^{5}$ & $>1100$ & $6.4 \times 10^{5}$ & Enterobacter \\
\hline Control 2 & $3.1 \times 10^{5}$ & $>1100$ & $1.5 \times 10^{5}$ & Enterobacter \\
\hline
\end{tabular}

Staphylococcus aureus has a special ability to colonize on the skin of patients with eczema and atopic dermatitis; and bacteria colonization is from major factors in aggravate skin lesions (15).

Haft-baradaran, et al. showed that $40 \%$ of the Iranian sunscreen creams, $73.3 \%$ of the imported products, and $43.3 \%$ of the formulated products at the time of purchase contained at least to one of the objectionable microorganisms of the samples showed contamination with Staphylococcus aureus, Pseudomonas aeroginosa. This study showed yeasts and molds contamination in evaluated creams too (3).

Keshavarz et al. study showed microbial contamination in $46 \%$ of the 135 moisturizing cream. The most contamination related was to Pseudomonas aeroginosa. Percentage of contamination to Pseudomonas aeroginosa, Staphylococcus aureus, coagulase-negative staphylococci, yeast, and mold were 35.5, 6.4, 26, 24.1, 6.4 and $1.6 \%$ respectively (14).

Lundov et al. reported $68 \%$ contamination in some moisturizing creams; $30 \%$ of these creams were contaminated to Staphylococcus aureus (16).

Behravan et al. showed that the percentage of contamination to Gram positive Bacilli, Staphylococcus aureus and nonEscherichia coli Gram-negative microorganisms in the used cosmetic creams, was 54, 38 and $8 \%$ respectively. This contamination in unused cosmetic creams was 38,25 and $0 \%$ respectively. This study proved that $17 \%$ of unused cream and $10 \%$ of used products were in the acceptable range of microbial content. According to Cosmetic, the requirements for cosmetic products set by the FDA, The cosmetic Toiletry and Perfumery Association Ltd. (CTPA) guidelines, and Cosmetic Toiletry and Fragrance Association Inc. (CTFA) guidelines and Cosmetic, Products with a high number of microorganisms or products containing pathogenic microorganisms would be considered as spoiled (6).

In the present study all samples (100\%) were contaminated with pathogenic microorganisms Enterobacter and counts of molds and yeasts (Table 1) were not in the acceptable range. Samples contained Staphylococcus aureus of pathogenic bacteria (14.29\%) was among samples of the informal market in Iran.

The important note in this study was high levels of contamination in the sunscreen creams before use and contamination in the creams was, in during manufacture and 
before reaching to the consumer which emphasis has on necessity to improve functional the quality assurance system in the manufacture process, packaging and adequate preservation and other factors involved in contamination (2).

\section{Conclusion}

The high microbial contamination was observed in Iranian and imported sunscreen creams. Microbial contamination of sunscreen creams is a potential health danger to consumers. It appears that it is necessary to inspect and supervise the products during manufacture and packaging and adequate preservation. It is strongly recommended to control and regulate cosmetics by health organization to ensure quality and safety of this type of products.

\section{References}

1. Birks S. Microbial risk in cosmetics and toiletries. Cleanroom Tech 2012;20:21-3.

2. Qazvini K, Safdari H. Evaluation of bacterial contamination in cosmetic eye product, before and after use. J Res Med Sci 2007;31:159-62.

3. Haft-baradaran B, Abedi D, Jalali M, Bagherinejad MR. Microbial quality survey of sunscreen products in Iranian market. Adv Biomed Res 2014;3:180.

4. Piu L, Juliano C, Minghetti P. Application of film method for microbial monitoring of cosmetic raw materials. J Appl Cosmetol 1996;14:155-61.

5. Claussen C. An investigation of bacterial growth in generic versus brand name lotions and shampoos over a 2 week period. SMU Bio J 2007:1-21. seminar.

6. Behravan J, Fazly Bazzaz BS, Malaekeh P. Survey of bacteriological contamination of cosmetic creams in Iran (2000). Int J Dermatol 2005;44:482-5.

7. Gagliardi L, De Orsi D, Dorato S. General review of official methods of analysis for cosmetics in different countries, In: Salvador A, Chisvert A (ed.): Analysis of cosmetic products. Elsevier, Netherland, 2007:4571 .

8. Lundov M, Moesby L, Zachariae C, Johansen J. Contamination versus preservation of cosmetics: A review on legislation, usage, infections, and contact allergy. Contact Dermatitis 2009;60:70-8.

9. Onurdağ FK, Özgen S, Abbasoğlu D. Microbiological Investigation of Used Cosmetic Samples. Hacettepe Uni J Fac Pharm 2010;30:1-16.

\section{Acknowledgment}

This article was written based on MSc. thesis of MA Narges Sedghi Sharif Abad, and was supported by a grant from the research council of the Mazandaran University of Medical Sciences.

\section{Conflict of interest}

The authors declared no potential conflict of interest with respect to the authorship, and/or publication of this study.
10. Lundov M, Zachariae C. Recalls of microbiologically contaminated cosmetics in EU from 2005 to May 2008. Int J Cosmet Sci 2008;30:471-4.

11. Okeke IN, Lamikanra A. Bacteriological quality of skin-moisturizing creams and lotions distributed in a tropical developing country. J Appl Microbiol 2001;91:922-8

12. Anelich LE, Korsten L. Survey of micro-organisms associated with spoilage of cosmetic creams manufactured in South Africa. Int J Cosmet Sci 1996; $18: 25-40$

13. United States Pharmacopeia (USP 30); 2007.

14. Keshtvarz M, Pourmand MR, Shirazi MH, Uosefi M, Hajikhani S. Microbial contamination of common cosmetic creams in Tehran. J Med Lab Sci 2014;8:959.

15. Gong JQ, Lin L, Lin T, Hao F, ZengFQ, Bi ZG, et al. Skin colonization by Staphylococcus aureus in patients with eczema and atopic dermatitis and relevant combined topical therapy: A double-blind multicentre randomized controlled trial. Br J Dermatol 2006;155:680-7.

16. Lundov M, Johansen J, Zachariae C, Moesby L. Creams Used by Hand Eczema Patients are often Contaminated with Staphylococcus aureus. Acta DermVenereol 2012;92:441-2. 